Thorax (1963), 18, 307

\title{
Diagnosis of the mitral lesion in patients with giant left atria
}

\author{
P. G. F. NIXON AND H. IKRAM \\ From the Department of Thoracic Surgery and Medicine, General Infirmary, Leeds
}

Aneurysmal dilatation of the left atrium occurs in mitral incompetence (Parsonnet, Bernstein, and Martland, 1946) and in mitral stenosis (Venner, 1954), and its presence may mask the underlying mitral lesion by modifying the left atrial pressure pulse (Fairley, 1961) and the indicator-dilution curve (Nixon and Snow, 1962).

The purpose of this communication is to describe the important clinical, haemodynamic, and surgical findings in 19 patients with gross enlargement of the left atrium, and to consider the diagnosis of the underlying mitral lesion.

\section{MATERIAL AND METHODS}

Nineteen patients with severe rheumatic mitral valvular disease and gross left atrial enlargement were selected for this study because the mitral valve had been examined at operation. Their ages ranged between 25 and 56 years, and 11 were women. None had hypertension or aortic valve disease. All were physically examined, and high or medium frequency phonocardiograms (Leatham, 1952) were recorded from the pulmonary and mitral areas. Twelve-lead electrocardiograms were obtained with a Sanborn TwinViso instrument (standardization: $1 \mathrm{~cm} .=1 \mathrm{mV}$ ). Six-foot penetrated postero-anterior chest radiographs were taken with the patient erect; the cardiothoracic ratio was noted, and the area of the left atrial shadow was measured with a planimeter. It is convenient to rank patients according to the area of the left atrial shadow, and it is not contended that the measurement is an exact index of the left atrial volume. The left atrial shadow was greater than $100 \mathrm{~cm}^{2}$ in every case (Figs. 1 to 3 ).

Right-heart and trans-septal left-heart catheterization was performed in the manner described by Nixon (1960). Pressure pulses and phonocardiograms were recorded. Ear-piece indicator dilution curves were obtained by injecting Coomassie blue dye into the right ventricular outflow tract or main pulmonary artery and into the left atrium. The appearance time between the right side of the heart and the ear was measured, and, where possible, the cardiac output and the ratio of least concentration to recirculation peak concentration (Wood and Woodward, 1957) were calculated from the curves obtained by the left atrial injection. The use of ear-piece curves for assessing valvular incompetence and the methods of calculating the results have been described previously (Nixon and Snow, 1962).

The mitral valve was examined at valvotomy (six cases) or open-heart operation (13 cases) by Mr. G. H. Wooler, and the long diameter of its orifice was estimated by inspection or palpation. In such severely disabled patients it was assumed that a small orifice caused severe obstruction and permitted little regurgitation, and a large orifice allowed great regurgitation but offered little obstruction. For the purposes of this study obstruction was diagnosed when the long diameter of the mitral orifice measured $1.5 \mathrm{~cm}$. or less, obstruction and regurgitation when it measured 1.6 to $2.5 \mathrm{~cm}$., and incompetence when it measured $2.6 \mathrm{~cm}$. or more.

\section{RESULTS}

Four patients had mitral obstruction and their atria measured 154, 170, 256, and $290 \mathrm{~cm} .^{2}$ Eight patients had obstruction and regurgitation and their atrial areas measured between 105 and 185 (average 140) $\mathrm{cm} .{ }^{2}$ Seven patients had incompetent valves and atria measuring 122 to 204 (average 153) $\mathrm{cm}^{2}$ (Figs. 1 to 3 ).

Mitral facies, peripheral cyanosis, and cold extremities were present in half the obstructed patients, in two of the eight cases of obstruction and regurgitation, and in one of the seven patients with incompetence.

The left ventricle was not palpable in three of the four cases of obstruction, but its apical thrust (Mackenzie, 1902) could be felt in the remainder. 

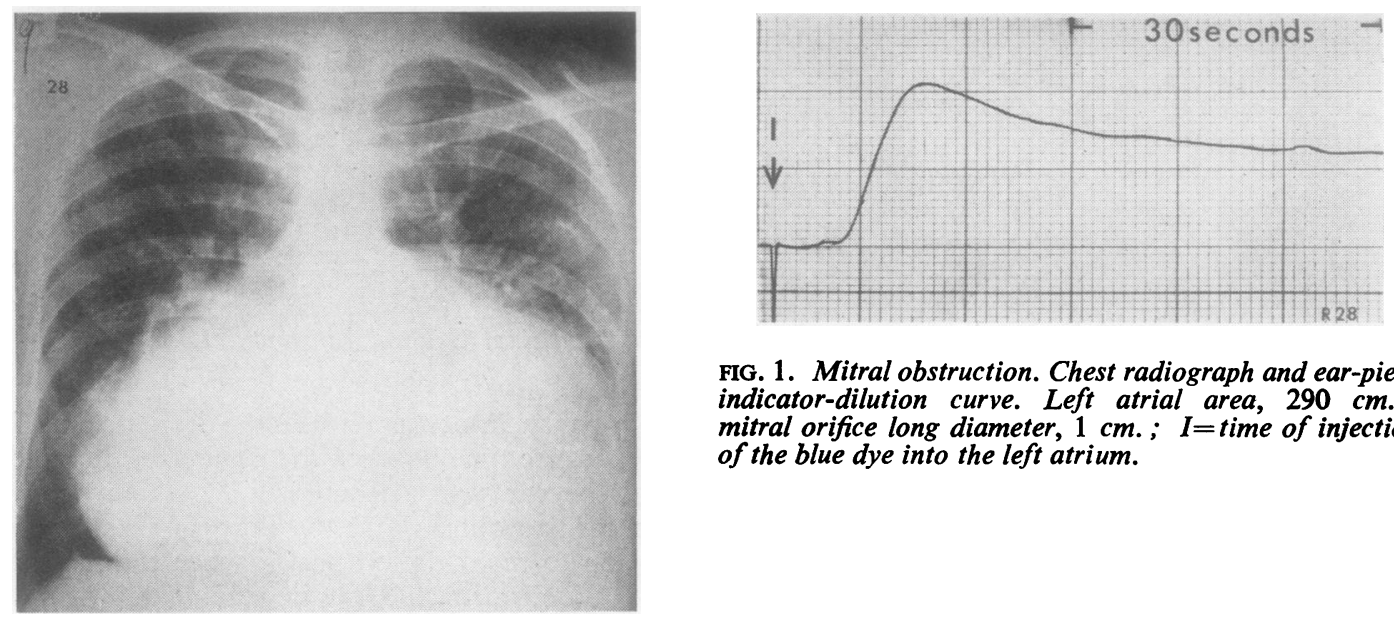

FIG. 1. Mitral obstruction. Chest radiograph and ear-piece indicator-dilution curve. Left atrial area, $290 \mathrm{~cm}^{2}$; mitral orifice long diameter, $1 \mathrm{~cm}$.; I=time of injection of the blue dye into the left atrium.
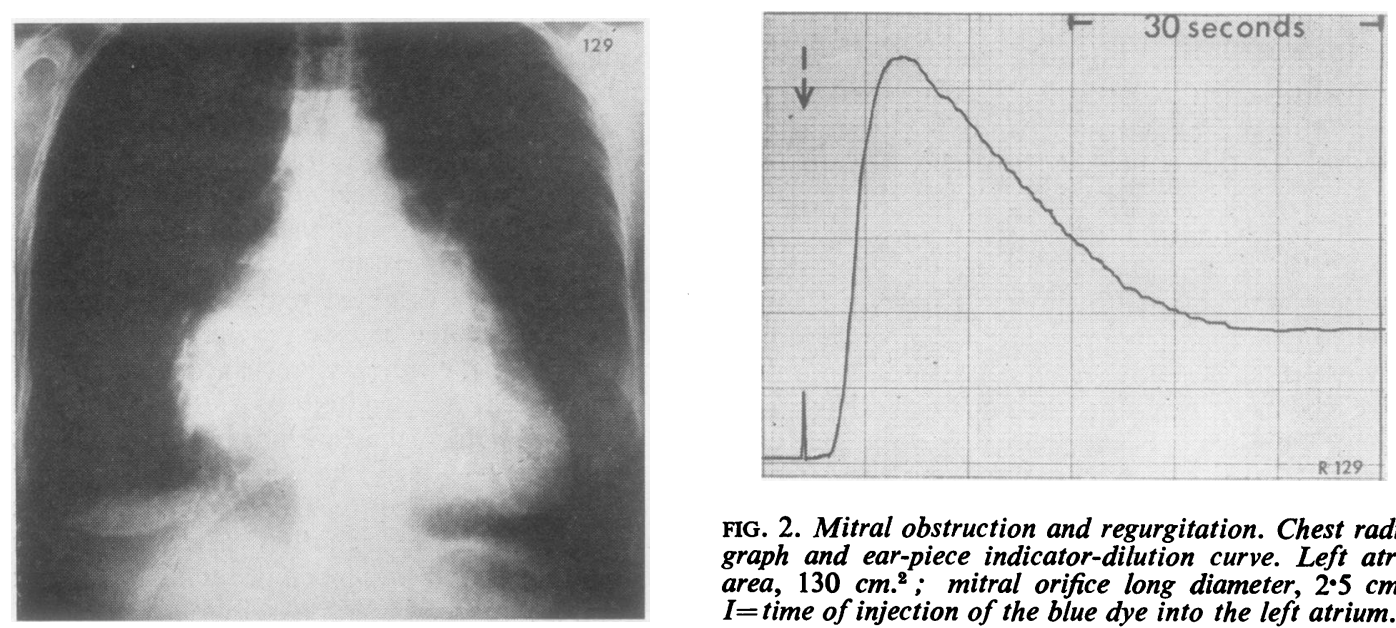

FIG. 2. Mitral obstruction and regurgitation. Chest radiograph and ear-piece indicator-dilution curve. Left atrial area, $130 \mathrm{~cm} .^{2}$; mitral orifice long diameter, $2.5 \mathrm{~cm}$.; $I=$ time of injection of the blue dye into the left atrium.
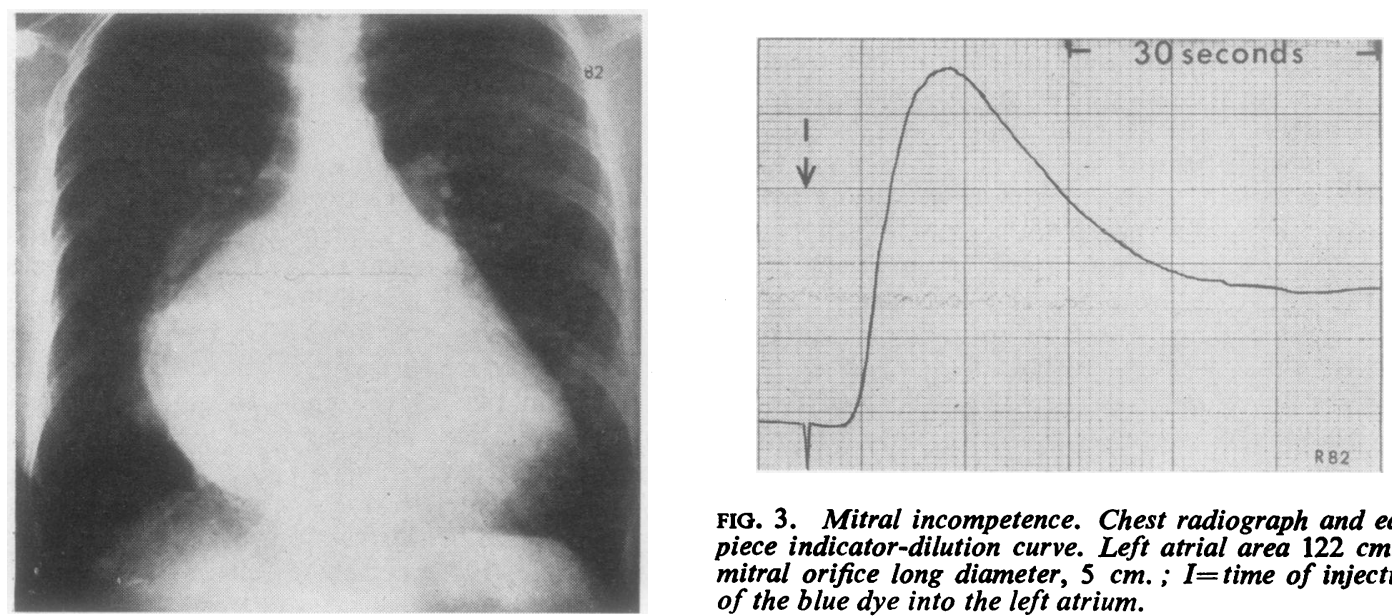

FIG. 3. Mitral incompetence. Chest radiograph and earpiece indicator-dilution curve. Left atrial area $122 \mathrm{~cm} .^{2}$; mitral orifice long diameter, $5 \mathrm{~cm}$.; I=time of injection of the blue dye into the left atrium. 


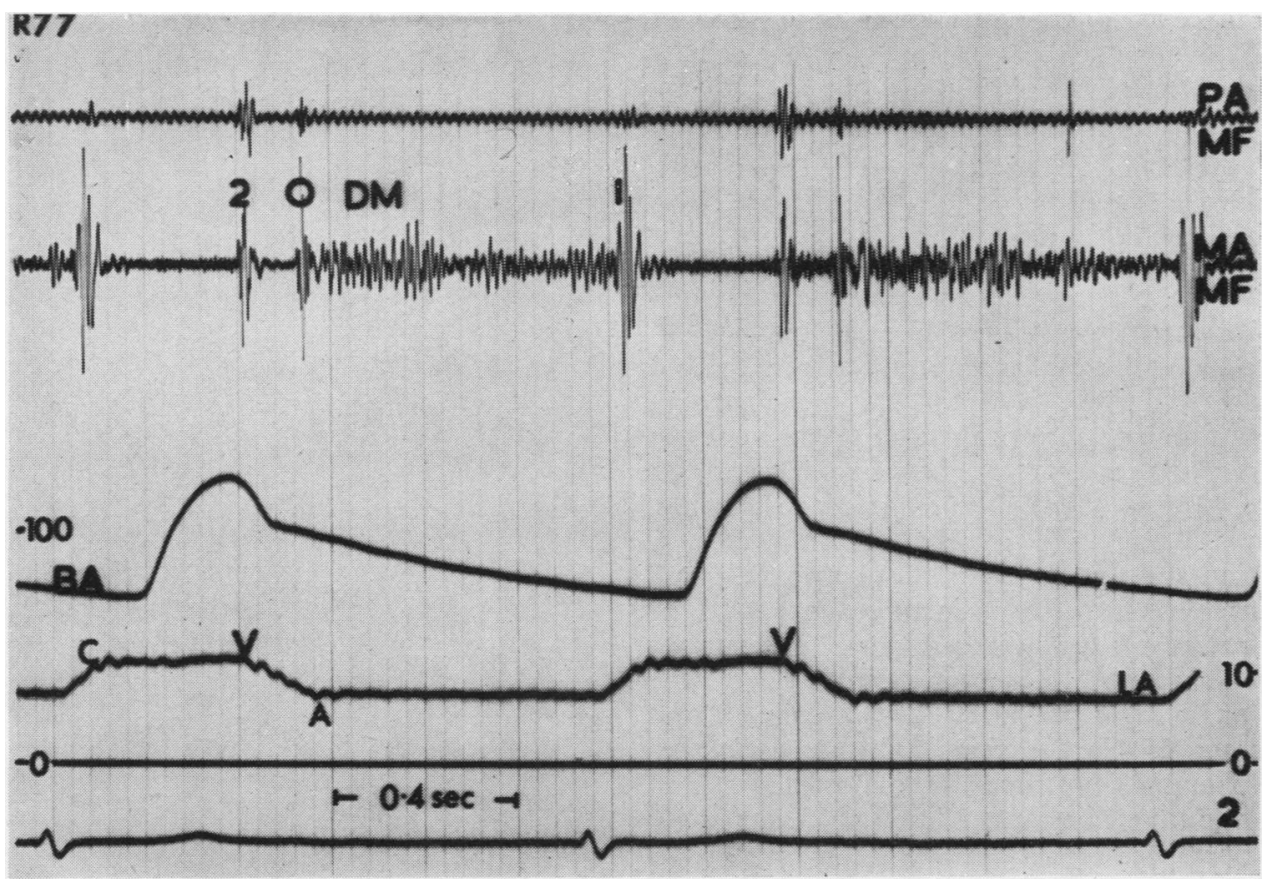

FIG. 4. Mitral obstruction. Pulmonary area medium-frequency (PA/MF) and mitral area medium-frequency (MA/MF) phonocardiograms; the brachial $(B A)$ and left atrial $(L A)$ pressure pulses; and the lead 2 electrocardiogram. 1, 2, $0=$ first and second heart sound, and mitral opening snap; DM=mitral diastolic murmur; $C, V=l e f t$ atrial ' $c$ ' and ' $v$ ' waves; $A=$ left atrial annular ascent point.

An apical pansystolic murmur was heard in every case save one patient with obstruction. The loudness of the murmur was graded 1 or 2 (Levine and Harvey, 1949) in the cases of obstruction, 2 or 3 in obstruction and regurgitation, and 3 or 4 in incompetence.

The mitral opening snap was present in every case.

The mitral diastolic murmur began quietly at the opening snap and rumbled throughout diastole without much fluctuation in loudness in the obstructed patients (Fig. 4), and in one with an orifice of $1.8 \mathrm{~cm}$. In the remainder it began loudly at the annular ascent point of the left atrial pulse and waned rapidly in size (Fig. 5).

Third heart sounds could not be detected when the mitral orifice measured $1.5 \mathrm{~cm}$. or less. All the patients with the larger orifices had third heart

FIG. 5. Mitral incompetence. Pulmonary area highfrequency $(P A / H F)$ and mitral area medium-frequency (MA/MF) phonocardiograms; the brachial $(B A)$ and left atrial $(L A)$ pressure pulses; and the lead 2 electrocardiogram; SM=mitral systolic murmur; $3=$ third heart sound; SW=left atrial stasis wave. Other symbols as for Fig. 4.

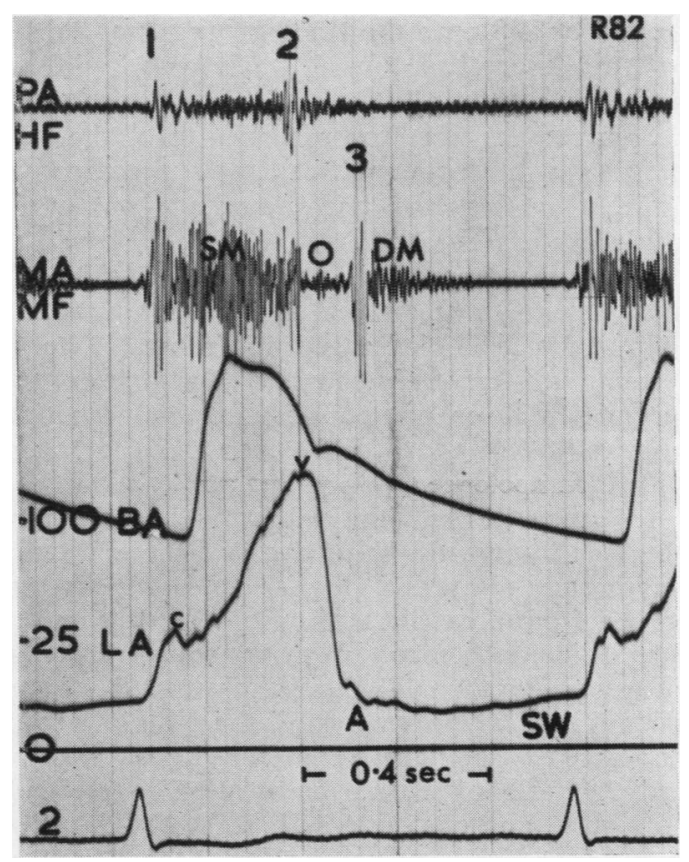


sounds, except for the one with an orifice of $1.8 \mathrm{~cm}$.

In the electrocardiograms the height of the tallest $R$ wave in V5 or V6 was added to the size of the deepest $S$ wave in V1 or V2. In the obstructed cases the result lay between 5 and 11 (average 8.5) $\mathrm{mm}$.; in obstruction and regurgitation, between 25 and 45 (average 38) $\mathrm{mm}$; ; and in incompetence, between 27 and 51 (average 40) $\mathrm{mm}$. Atrial fibrillation was present in every patient.

The cardio-thoracic ratios lay between 65 and $89 \%$, with an average of 70 to $72 \%$ in each group.

The systolic pressure peak in the right ventricle or pulmonary artery lay between 17 and 88 (average 42) $\mathrm{mm}$. $\mathrm{Hg}$ in the cases of obstruction, between 25 and 65 (average 41) $\mathrm{mm}$. $\mathrm{Hg}$ in obstruction and regurgitation, and between 34 and 78 (average 56) $\mathrm{mm} . \mathrm{Hg}$ in incompetence.

The peaks of the left atrial ' $v$ ' waves were measured in $\mathrm{mm}$. $\mathrm{Hg}$ from the level of the sternal angle. The pressures lay between 10 and 43 (average 23) in the cases of obstruction, between 15 and 43 (average 27) in obstruction and regurgitation, and between 27 and 55 (average 40) in incompetence.

Left atrial stasis waves (Nixon and Wooler, 1961) were not found in the obstructed cases. They were present in five of the eight patients with obstruction and regurgitation, but were absent in the one who had a quietly-beginning diastolic murmur, no third heart sound, and an orifice measuring $1.8 \mathrm{~cm}$; the tracings were not suitable for the identification of stasis waves in two patients.

Stasis waves were recorded in five of the seven patients with incompetence; their presence was doubtful in the sixth, and the tracing was unsuitable in the seventh.

The appearance time at the ear of blue dye injected into the right ventricular outflow tract or pulmonary artery averaged 15 (range 13 to 18 ) seconds in the obstructed cases, 10 (range 6 to 14) seconds in those with obstruction and regurgitation, and 8.5 (range 7 to 12 ) seconds in the patients with incompetence.

In every patient the indicator-dilution curve obtained by injecting blue dye into the left atrium was grossly abnormal (Figs. 1 to 3 ). A curve recorded from a patient with mitral stenosis and tricuspid incompetence, in whom the left atrium measured less than $100 \mathrm{~cm} .{ }^{2}$, is illustrated for comparison (Fig. 6). In most cases the dip between the primary peak and the recirculation peak was lost, and the ratio of least concentration to recirculation peak concentration (CL/CR) could not be

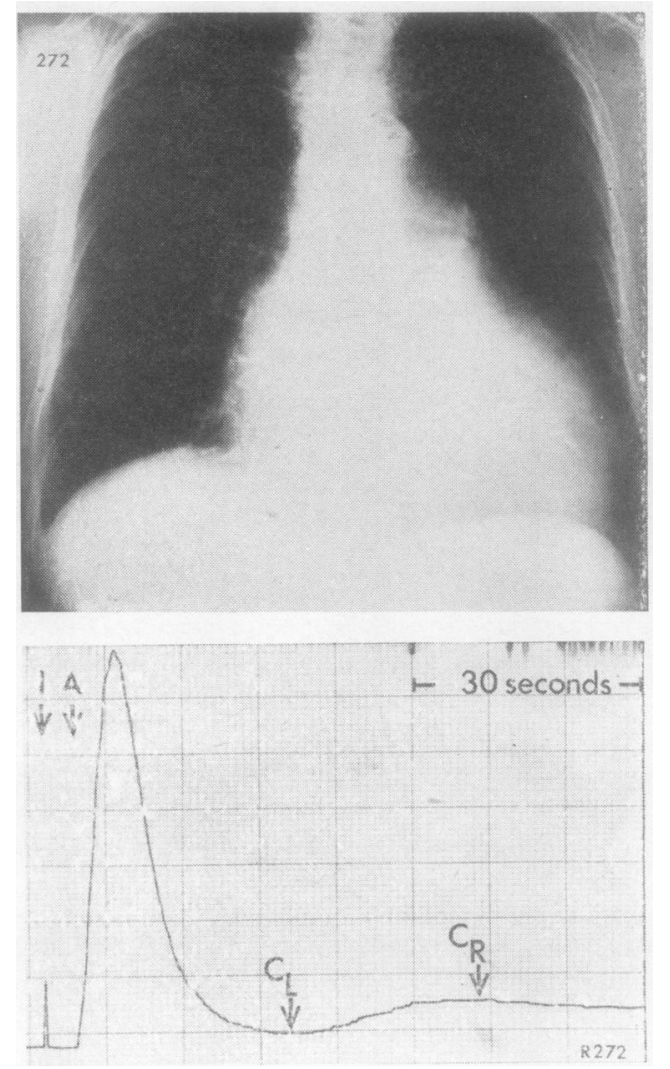

FIG. 6. Mitral obstruction. Chest radiograph and earpiece indicator-dilution curve (injection into left atrium). Left atrial area less than $100 \mathrm{~cm} .^{2}$ Mitral orifice long diameter, $0.5 \mathrm{~cm} . ; \quad I=$ time of injection of the blue dye into the left atria; A=time of appearance of the blue dye at the ear ; $C L=$ least concentration ; $C R=$ recirculation peak concentration.

measured. Where it could be calculated the result was 0.9 in a case of obstruction, $0.7,0.9$, and 0.9 in obstruction and regurgitation, and 0.7 and 0.9 in incompetence.

The cardiac index was estimated in two cases of obstruction and found to be 1.65 and 1.5 . In obstruction and regurgitation the cardiac index lay between 1.7 and 2.9 (average $2 \cdot 1$ ), and in incompetence between 1.8 and 3.6 (average 3.0 ).

\section{DISCUSSION}

This study supports the belief that aneurysmal dilatation of the left atrium is usually associated with severe mitral incompetence (Parsonnet et al., 1946; Daley and Franks, 1949; Wood, 1954), but confirms the observation that it can occur in cases 
of severe obstruction (Venner, 1954; Goodwin, Hunter, Cleland, Davies, and Steiner, 1955; Kent, Fisher, Ford, and Neville, 1956; McDonald, Dealy, Rabinowitz, and Dexter, 1957). It follows that gross enlargement of the left atrium is not in itself diagnostic of the underlying valvular lesion.

Gross left atrial enlargement might be expected to modify the physical signs and the results of investigations in mitral valvular disease because it alters the position of the remainder of the heart and disturbs the volume and pressure relations between the left atrium and ventricle. This series of patients is small. If it is representative, the cases of obstruction can be identified by the following features: the diastolic murmur begins close to the opening snap and changes little in loudness as it rumbles throughout diastole; there is no third heart sound; and left atrial stasis waves do not occur. An apical pansystolic murmur may be present or absent, and the apex of the left ventricle may or may not be palpable. In obstruction and regurgitation and in severe incompetence the diastolic murmur begins with loud vibrations close to the annular ascent point of the left atrial pulse and wanes rapidly (Nixon, 1961); a third heart sound is present; and a left atrial stasis wave can be recorded in the majority of patients. An apical pansystolic murmur is prominent, and the apex of the left ventricle is easily palpable. The differences between obstruction and regurgitation on the one hand, and incompetence on the other, appear to be differences of degree rather than differences of kind.

In these respects the patients selected for their gross left atrial size resemble other cases of severe mitral disease (Nixon, 1961; Nixon and Wooler, 1960).

The right ventricular and the left atrial ' $v$ ' peak pressures tended to increase with the severity of the incompetence in this series, but there was too much variation within the groups for the measurements to have a diagnostic value.

The observation that the indicator-dilution curves were grossly distorted in the absence of severe regurgitation supports Conn's (1959) conclusion that the left atrial residual volume has an important part to play in shaping the curve (Nixon and Snow, 1962). There was little overlap between the prolonged indicator-dilution appearance times of the obstructed cases and the shorter appearance times of the remainder, and this finding agrees with the conclusions of Shillingford (1958). The ratio CL/CR was valueless. The cardiac index tended to increase in proportion to the severity of the regurgitation. When the area of the left atrial shadow is less than $100 \mathrm{~cm} .^{2}$, the ratio CL/CR appears to have a great diagnostic value, and the cardiac output is smaller in the more
FIG. 7. The electrocardiogram in a case of mitral obstruction and aneurysmal dilatation of the left atrium.

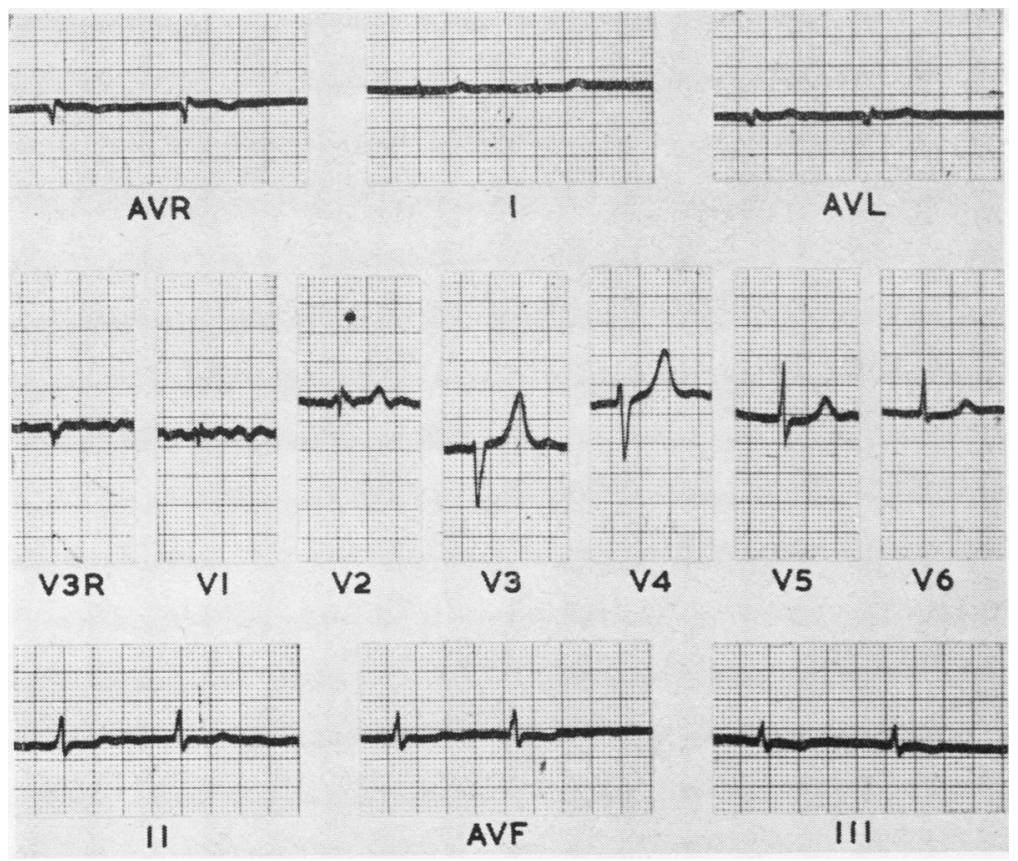


severely incompetent cases (Nixon and Snow, 1962).

The electrocardiogram distinguished the obstructed patients from the remainder in the cases reported here; nevertheless in patients with left atria of less than $100 \mathrm{~cm} .^{2}$ it is not rare to find severe mitral incompetence unaccompanied by cardiographic evidence of left ventricular hypertrophy. In 1957 Grant suggested that the abnormal location of the ventricles in the giant left atrial syndrome might cause the QRS complexes of the left lateral praecordial leads to resemble the QRS complexes of left ventricular hypertrophy, but it did not do so in the cases of obstruction reported here (Fig. 7).

\section{SUMMARY}

Nineteen patients with aneurysmal dilatation of the left atrium were examined, investigated, and treated surgically. Reference to the mitral diastolic murmur, the third heart sound, and the electrocardiogram allowed the four patients with severe obstruction to be distinguished from the eight with obstruction and regurgitation, and from the seven with severe incompetence. There was a tendency for the right ventricular and left atrial ' $v$ ' peak pressures and the cardiac indices to increase in proportion to the severity of the regurgitation, and the indicator-dilution appearance times were longest in the obstructed cases. Left atrial stasis waves were not found when the mitral orifice measured $1.5 \mathrm{~cm}$. or less, but they occurred in the majority of patients with larger orifices.

The authors are deeply indebted to Mr. G. H. Wooler and Professor R. E. Tunbridge for making this study possible. They are grateful to Mr. H. M. Snow.
Mr. R. Addyman, Miss V. Tuke, and Mrs. M. Williams for assistance. The Board of Governors of the United Leeds Hospitals, the Trustees of the Nuffield Foundation, and private benefactors have provided financial help.

\section{REFERENCES}

Conn, H. L. (1959). Use of indicator-dilution curves in the evaluation of acquired heart disease. Progr. cardiovasc. Dis., 2, 166.

Daley, R., and Franks, R. (1949). Massive dilatation of the left auricle. Quart. J. Med., 18, 81.

Fairley, K. F. (1961). The influence of atrial size and elasticity on the left atrial pressure tracing. Brit. Heart J., 23, 512.

Goodwin, J. F., Hunter, J. D., Cleland, W. P., Davies, L. G., and Steiner, R. E. (1955). Mitral valve disease and mitral valvotomy. Brit. med. J., 2, 573 .

Grant, R. P. (1957). Clinical Electrocardiography, The Spatial Vector Approach. McGraw-Hill, New York.

Kent, E. M., Fisher, D. L., Ford, W. B., and Neville, J. F. (1956). Mitral valve surgery and left heart catheterization in giant left atrium. Arch. Surg., 73, 503.

Leatham, A. (1952). Phonocardiography. Brit. med. Bull., 8, 333.

Levine, S. A., and Harvey, W. P. (1949). Clinical Auscultation of the Heart. Saunders, Philadelphia.

Mackenzie, J. (1902). The Study of the Pulse. Young J. Pentland, Edinburgh and London.

McDonald, L., Dealy, J. B., Rabinowitz, M., and Dexter, L. (1957) Clinical, physiological and pathological findings in mitra stenosis and regurgitation. Medicine (Baltimore), 36, 237.

Nixon, P. G. F. (1960). The transeptal approach to the left atrium in mitral regurgitation. Thorax, 15, 225.

(1961). The third heart sound in mitral regurgitation Brit. Heart J., 23, 677.

valvular disease. Ibid., 24, 637.

, and Wooler, G. H. (1960). Clinical assessment of mitral orifice in patients with regurgitation. Brit. med. J., 2, 1122.

- - - (1961). Rapid left ventricular filling and stasis in mitral regurgitation. Brit. Heart J., 23, 161 .

Parsonnet, A. E., Bernstein, A., and Martland, H. S. (1946). Massive left auricle with special reference to its etiology and mechanism. Amer. Heart J., 31, 438.

Shillingford, J. (1958). Simple method for estimating mitral regurgitation by dye dilution curves. Brit. Heart J., 20, 229.

Venner, A. (1954). Massive left atrium and mitral valvotomy. Brit med. J., 1, 1359 .

Wood, P. (1954). An appreciation of mitral stenosis. Ibid., 1, 1051.

Wood, E. H., and Woodward, E. (1957). A simple method for differentiating mitral regurgitation from mitral stenosis by indicator-dilution curves. Proc. Mayo Clin., 32, 536. 